Revista

\title{
Multi-Ensayos
}

Vol. 6, núm. 11

ISSN: 2412-3285

https://multiensayos.unan.edu.ni

DOI: https://doi.org/10.5377/multiensayos.v6i11.9290

\section{Prácticas de laboratorio: la antesala a la realidad}

\section{Laboratory practices: the anteroom to reality \\ Edwin Antonio Reyes Aguilera ${ }^{1}$}

Recibido: 25 de octubre de 2019. Aceptado: 14 de enero de 2020

\section{RESUMEN}

Las prácticas de laboratorio constituyen un estado efectivo de aprender a hacer, razonar, interactuar, debatir, poner en común ideas, puntos de vista y por supuesto poder transformar la realidad. Constituyen en efecto, un mero acercamiento a la realidad que el futuro ingeniero en energías renovables encontrará, constituye una ruta abierta entre la teoría y la práctica de manera que a través de la experimentación la teoría pueda ser comprobada o por el contrario falseada. En el presente ensayo se realiza una pequeña reflexión sobre la importancia de las prácticas de laboratorio y el rol del docente en el desarrollo de estas, finalmente se presenta parte de la experiencia como docente hacedor de prácticas de laboratorio en diferentes asignaturas y el impacto en los estudiantes egresados.

Palabras clave: prácticas de laboratorio; energías renovables; teoría; práctica.

\section{ABSTRACT}

Laboratory practices are a productive activity to learn how to do, reason, interact, debate, share ideas, views, and of course, be able to transform reality. Laboratory practices constitute a mere approach to the reality that the future renewable energy engineer will find and constitute an open route between theory and practice so that through experimentation, theory can be proven or, on the contrary, rejected. This essay presents a small reflection on the importance of laboratory practices and the role of teachers in their development. Finally, it describes part of the experience as a teacher conducting lab practices ant its different impacts on graduate students.

Keywords: laboratory practices; renewable energies; theory; practice.

1 Docente UNAN-Managua/FAREM-Estelí. Correo electrónico: edwinra11@yahoo.es. (c) 2020 Revista Multi-Ensayos. 


\section{INTRODUCCIÓN}

La responsabilidad de la formación de profesionales de ingeniería en la actualidad, implica la conformación de pertinentes competencias que involucran la integración de saberes, habilidades y actitudes generales y específicas. La vinculación entre la teoría y la práctica es uno de los problemas que más esfuerzo conlleva en el desarrollo de los programas de asignatura.

Las prácticas de laboratorio vienen hacer como una herramienta en el aprendizaje para los estudiantes, ya que brindan la posibilidad de entender cómo se construye el conocimiento dentro de la comunidad científica, además aportan una mejor comprensión teórica en diversos contenidos aclarando con mayor facilidad las dificultades presentada por los estudiantes, permitiendo cuestionar sus saberes y confrontarlos con la realidad. Además, el estudiante pone en juego sus conocimientos previosy los verifica mediante las prácticas. La actividad experimental no solo debe ser vista como una herramienta de conocimiento si no como un instrumento que promueve los objetivos conceptuales, procedimentales y actitudinales que debe incluir cualquier dispositivo pedagógico (Osorio, 2004, págs. 7-10) .

En la carrera de Ingeniería en Energías Renovables de la UNAN Managua - FAREM Estelí se ha dotado de un laboratorio de electricidad y electrónica, gracias a convenios establecidos con la Universidad Carlos Tercero de Madrid España de modo que nos ha permitido no limitarnos a la teoría únicamente, sino que, se ha dinamizado a través de la vinculación del discurso propio dicho en clase con la práctica, en consecuencia, se genera alta motivación en los estudiantes. El interés principal es promover el aprendizaje básico sobre las energías renovables, de forma manipulativa, sobre todo porque consideramos que el aprendizaje así conseguido, es más significativo y duradero.

\section{DESARROLLO}

\section{La importancia de la practica en la ingeniería en energías renovables}

El aprendizaje significativo hace énfasis en estrategias metodológicas de construcción de conocimientos, en el saber hacer, que necesita para lograrse del saber y del saber ser como condiciones sine qua non. Las estrategias que sobresalen en este tipo de aprendizaje son aquellas que, además de presentar un producto, demandan un fuerte componente procedimental-actitudinal capaz de provocar la Meta cognición del aprendiz. Es decir, favorecen el procesamiento profundo de información, la estructuración lógica y adecuada de ésta, y finalmente, crean recuerdos más efectivos sobre lo aprendido (UNAN Managua, 2011).

Las prácticas de laboratorio constituyen una condición válida de conocer y transformar la realidad, es así que, más allá de los conocimientos teóricos aportados en las sesiones de clase por los docentes, las practicas permiten construir conocimiento cercano a lo que el estudiante encontrara en la realidad, es decir, en su vida profesional; movilizarlo e integrarlo con otros, para luego, desarrollar habilidades, destrezas y actitudes de forma significativa. 
La experimentación tiene un rol muy importante como una actividad que acuerda la interacción entre lo natural (fenómeno) y lo social (el experimentador y pares), ligado a un espacio o sitio determinado, comúnmente, conocido como laboratorio (Marín Quintero, 2010).

Es en el laboratorio donde el experimentador realiza un proceso de intervención al objeto de estudio a través de procedimientos, técnicas, instrumentos y aparatos, haciendo uso de procesos cognitivos y manipulativos e incluso de matematización cuando se incluyen procesos de medición de datos cuantitativos, con la intención de producir determinadas reacciones (cambios empíricos). Tiene un rol muy importante como parte integrante de la constitución de la propia teoría, es decir, la integración de la teoría y la experimentación es inevitable, y por ninguna consideración la cantidad de experimentación, por sí misma, y aislada de la teoría puede llegar a originar un nuevo concepto (Marín Quintero, 2010).

La experimentación en laboratorio es una gran oportunidad donde el estudiante puede el mismo verificar aquellos conceptos teóricos recibidos y memorizados en el aula de clases, estas prácticas constituyen el primer contacto con la realidad que el futuro ingeniero en energías renovables enfrentará.

En la carrera de ingeniería en energías renovables se ha tomado la iniciativa de que los docentes que imparten clases de la especialidad realicen prácticas de laboratorio, de tal forma que se establezca un ambiente de aprendizaje significativo, duradero y de calidad y por esta razón, este tipo de formación alcance un papel predominante que refleje toda la teoría trabajada en el aula y no dejar esta tarea y responsabilidad a las empresas.

La mayoría de las asignaturas del pensum académico de esta carrera sugiere la realización de prácticas de laboratorio. En algunas asignaturas no se realiza practicas debido a no contar con la infraestructura adecuada. Existen dos asignaturas en el cual los laboratorios conforman una materia en sí, (Laboratorio de mediciones eléctricas y electrónicas y Laboratorio de fluidos) independiente de la calificación de la clase teórica a la cual está asociada.

Considero que las prácticas de laboratorio forman parte del primer contacto que el estudiante tiene con la realidad y deben estar orientadas a lo que encontrará en el mundo real. Debemos enseñar mediante la metodología aprender haciendo porque es de este modo que el futuro ingeniero resolverá sus dificultades, problemáticas o necesidades específicas una vez este dentro del mundo laboral.

El laboratorio constituye el espacio dinámico, adecuado y de interés de los estudiantes que desde el primer año de su carrera sienten la necesidad de conocer, luego que ya inician sus primeras practicas se dan cuenta que es aquí, donde deben desarrollar su habilidad creativa, propositiva e interpretativa a través del contacto con los dispositivos eléctricos y electrónicos, fotovoltaicos, térmicos y fluidos.

El desarrollo de prácticas de laboratorio incita y provoca la curiosidad de los estudiantes, ayuda y beneficia el aprendizaje de las energías renovables en los estudiantes, pues le permite debatir los conocimientos previos teóricos adquiridos en el aula de clase y verificarlos con la realidad. Las clases teóricas acompañadas 
de la experimentación constituyen una herramienta poderosa que aporta al desarrollo de habilidades y destrezas que seguramente les exigirá el campo laboral.

Las prácticas de laboratorio realizadas han servido a nuestros egresados en su campo laboral, algunos están trabajando en empresas de servicios eléctricos, en las que ponen en práctica lo aprendido en el laboratorio y para ellos no representa un mundo desconocido, ellos reconocen la importancia de haber realizado laboratorios de sus diferentes asignaturas y particularmente las elaboradas en las asignaturas de circuitos eléctricos, maquinas eléctricas, mediciones eléctricas y electrónicas, energía solar fotovoltaica y mecánica de fluidos.

Como docente formado especialmente para desarrollar estas prácticas de laboratorio puedo afirmar categóricamente que mediante este tipo de metodología el estudiante adquiere seguridad, sabe que se le está acercando la teoría con la realidad, que, al estar egresado experimentará, conocerá y será parte de la solución de los problemas. Se le ofrece un abanico de posibilidades de razonamiento, autonomía, de desarrollo de habilidades y destrezas con equipos e instrumentos de medición.

\section{¿Cuál sería la actitud del docente de laboratorio?}

La implementación de las prácticas de laboratorio implica un proceso de enseñanza-aprendizaje facilitado y regulado por el docente, el cual debe organizar temporal y espacialmente ambientes de aprendizaje para ejecutar etapas estrechamente relacionadas que le permitan a los estudiantes, realizar acciones psicomotoras y sociales a través del trabajo colaborativo, establecer comunicación entre las diversas fuentes de información, interactuar con equipos e instrumentos y abordar la solución de los problemas desde un enfoque interdisciplinar-profesional (Cardona Buitrago, 2013).

De acuerdo a (Cruz \& Valencia, 2005) La actitud del profesor de laboratorio es importante y su participación proactiva en el mismo le exige una mayor preparación, ya que estará expuesto a gran cantidad de interrogantes y posiblemente no podrá responder a alguno de ellos, pero debe estar en capacidad de poder sugerir alternativas que al ser implementadas den un resultado positivo al grupo de trabajo. No deberá ser autoritario ni incidir en las decisiones que tomen los estudiantes porque puede caer en la coartación de actividades creativas de los discentes.

En este sentido se ha orientado desde la coordinación de la carrera que, los docentes que realicen laboratorios deben entregar al estudiante el guion de laboratorio con anticipación de tal manera, que vayan haciendo sus averiguaciones y sepan concretamente que es lo que harán en el laboratorio. A sí mismo el docente deberá ensayar la practica con anticipación para que, pueda estar preparado para dar asistencia a los grupos de trabajo, aclarar dudas y en la mayoría de los casos revisar los montajes experimentales de los estudiantes con el fin de, que los resultados obtenidos sean los esperados según los objetivos planteados, pero haciendo énfasis en el aprendizaje autónomo del estudiante y dejarle claro que resolver problemas es parte activa de su aprendizaje. 
La experiencia de desarrollar prácticas de laboratorio me ha permitido identificar ahora con detenimiento etapas que se desarrollan dentro de las prácticas. Las etapas son en función de, antes de la experimentación, durante la experimentación y después de la experimentación.

a) Antes de la experimentación

Se hace entrega del guion de laboratorio a los estudiantes con dos o tres días de anticipación de manera que estos lean, indaguen sobre la temática a experimentar, luego al momento de inicio de la práctica se hace una introducción de lo que el estudiante realizará, se recomienda que asuman con disciplina, atención y responsabilidad el trabajo colaborativo de lo que posiblemente se enfrenten una vez estén en el campo laboral.

En algunos casos el estudiante debe de llevar previamente elaborada la parte teórica que se encuentra en el guion, que le permita poder confrontar estos cálculos con lo experimentado.

b) Durante la experimentación

Antes de dar inicio al proceso experimental, se hace una lectura del guion de laboratorio e introduciendo preguntas diagnósticas y dirigidas que pueden ser simples o complejas sobre el diseño experimental a modo de sondeo, comunicándoles el tiempo que disponen, los componentes, equipos, materiales y haciendo especial énfasis en la seguridad de cada uno dentro del laboratorio.

Durante el proceso de experimentación se asiste con regularidad cada grupo de trabajo colaborando con la correcta y segura instalación de los montajes, conocer las dificultades, hacerles ver que el trabajo es en equipo, deben apoyarse entre ellos y no llamar al docente a la menor dificultad. Los estudiantes deben realizar sus montajes, gerenciar el tiempo que disponen para la experimentación, delegarse responsabilidades dentro del grupo de trabajo, obtener los datos experimentales de sus mediciones u observaciones, realizar más de dos repeticiones para obtener datos fiables, coherentes con los cálculos teóricos previamente realizados que les permita comprobar o falsear una teoría.

Al finalizar la práctica se les recuerda la elaboración y entrega del informe de la práctica, se hace recepción de los materiales, equipos que formaron parte de la experimentación, se hace mención de la limpieza del laboratorio.

\section{c) Después de la experimentación}

La entrega deben hacerla en la sesión de clase posterior al laboratorio realizado, esto con la correcta interpretación de los datos del experimento real y que explique las diferencias encontradas con los cálculos teóricos mediante un razonamiento crítico, interpretando correctamente los datos, pudiendo llegar a conclusiones de tal manera que puedan debatir, poner en común ideas y resolver las cuestiones planteadas en el guion de laboratorio. 


\section{CONCLUSIÓN}

Las prácticas de laboratorio constituyen una metodología de enseñanza-aprendizaje que permite que los egresados una vez en el campo laboral puedan desenvolverse sin temor a las actividades que realizaran. Al final gana el vínculo: universidad, docente, estudiante y la empresa.

El desarrollo de las practicas provoca e incita al estudiante a interesarse en las asignaturas de tal manera que el aprendizaje adquirido sea el idóneo en la promoción de significativos resultados y motivación en ellos. Como decía Aristóteles: "Lo que tenemos que aprender a hacer, lo aprendemos haciéndolo".

De acuerdo a la experiencia adquirida puedo resaltar que, las prácticas de laboratorio son una antesala a la realidad que el estudiante deberá asumir con disciplina, compromiso y responsabilidad. Sin embargo, como docente estoy claro que se requiere de más tiempo y preparación que las conferencias convencionales; el resultado es rico, placentero y el grado de autorrealización que me reporta es inconmensurable.

\section{REFERENCIAS}

Cardona Buitrago, F. E. (2013). LAS PRÁCTICAS DE LABORATORIO COMO ESTRATEGIA DIDÁCTICA. Tesis de grado, Universidad del Valle, Santiago de Calí. Recuperado el 29 de Octubre de 2019, de http:// bibliotecadigital.univalle.edu.co/bitstream/10893/6772/1/CD-0395428.pdf

Cruz, J. C., \& Valencia, J. F. (2005). La formación práctica del ingeniero electrónico en el laboratorio. Revista Cientifica Guillermo de Ockham, III(1), 115-130. Recuperado el 24 de Octubre de 2019, de https:// www.redalyc.org/articulo.oa?id $=105316842007$

Marín Quintero, M. (2010). El trabajo experimental en la enseñanza de la quimica en contexto de resolución de problemas. Revista EDUCyT, I, 37-52. Recuperado el 25 de Octubre de 2019, de http://bibliotecadigital.univalle.edu.co/bitstream/10893/7553/1/3.pdf

Osorio, Y. W. (2004). El experimento como indicador de aprendizaje. Boletín PPDQ(43), 7-10.

UNAN Managua. (2 de Septiembre de 2011). Modelo Educativo, Normativa y Metodología para la Planificación Curricular. 39. managua, Nicaragua. Recuperado el 28 de Octubre de 2019, de http:// pagines.uab.cat/unan_uab_innovadocencia/sites/pagines.uab.cat.unan_uab_innovadocencia/ files/Modelo_Educativo19_de_septiembre.pdf 